\title{
Understanding Link Dynamics in Wireless Sensor Networks with Dynamically Steerable Directional Antennas
}

\author{
Thiemo Voigt ${ }^{1,2}$, Luca Mottola ${ }^{2,3}$, Kasun Hewage ${ }^{1}$ \\ \{thiemo,luca\}@sics.se, kasun.hewage@it.uu.se \\ 1 Uppsala University, Sweden \\ 2 Swedish Institute of Computer Science (SICS), Kista, Sweden \\ ${ }^{3}$ Politecnico di Milano, Italy
}

\begin{abstract}
By radiating the power in the direction of choice, electronicallyswitched directional (ESD) antennas can reduce network contention and avoid packet loss. There exists some ESD antennas for wireless sensor networks, but so far researchers have mainly evaluated their directionality. There are no studies regarding the link dynamics of ESD antennas, in particular not for indoor deployments and other scenarios where nodes are not necessarily in line of sight. Our long-term experiments confirm that previous findings that have demonstrated the dependence of angleof-arrival on channel frequency also hold for directional transmissions with ESD antennas. This is important for the design of protocols for wireless sensor networks with ESD antennas: the best antenna direction, i.e., the direction that leads to the highest packet reception rate and signal strength at the receiver, is not stable but varies over time and with the selected IEEE 802.15.4 channel. As this requires protocols to incorporate some form of adaptation, we present an intentionally simple and yet efficient mechanism for selecting the best antenna direction at run-time with an energy overhead below $2 \%$ compared to standard omni-directional transmissions.
\end{abstract}

\section{Introduction}

For many wireless sensor network (WSN) applications, reliability and energyefficiency are among the most critical issues. Electronically-switched directional (ESD) antennas are able to steer the radiated power in specific directions that can be selected via software. By concentrating the radiated power in one direction, ESD antennas enable lower contention and extended range without additional energy consumption. Lower contention avoids packet loss and hence reduces the need for retransmissions, which saves energy. While currently ESD antennas are not frequently used in WSNs, we have demonstrated their potential by showing that only slight modifications to Contiki Collect [1], a traditional CTP-like convergecast protocol, make it efficient for ESD antennas and lead to higher packet yield at lower duty cycle [2]. 
Recently, researchers have designed several ESD and other types of directional antennas for WSNs [3-6]. Most measurements with these antennas, however, have only assessed the directionality of the antenna, showing that the received signal strength of nodes that are in the direction in which the antenna radiates its maximum power is indeed higher than that of nodes at other locations. These measurements have been short-lived and were mostly conducted outdoors in open space where the receiving nodes have been placed in line of sight (LOS) of the transmitter. While these measurements fulfil the purpose of demonstrating the main property of ESD antennas, i.e., directionality, they provide little insight on the link dynamics.

The goal of our study is to gain a more thorough understanding of the dynamics of link quality that is needed when developing protocols for networks with ESD antennas. In the absence of studies like the one we present in this paper, researchers made implicit assumptions about the stability of links $[7,8]$. Our results show that, for example, many of the existing mechanisms to select the preferred antenna configuration need to be complemented with mechanisms that continuously evaluate if the current selection of active antenna directions is still the preferred configuration, or if adaption is needed.

In this paper, we therefore conduct long-term indoor experiments with SPIDA, an ESD antenna designed specifically for WSNs [5]. Via a simple software API, the SPIDA antenna can be configured to steer the radiated power in six different directions, in addition to the possibility of performing traditional omnidirectional transmissions. Our experiments also include nodes that are not in LOS of the transmitter.

Our experimental results show that in particular in non-LOS conditions, the best antenna direction, i.e., the direction that leads to the highest packet reception rate and signal strength at the receiver, is not necessarily related to the physical position of the receiver with respect to the transmitter. It rather depends on multi-path effects. Furthermore, the best antenna direction is not stable but varies over time which requires protocols to adapt. Since multi-path induced fading varies with frequency, the antenna direction that performs best on one channel does not necessarily perform well on another channel. These results confirm that previous findings that have shown that channel frequency and changes in the environment that happen over time have an affect on the best reception angle [9] also hold for directional transmissions with ESD antennas.

Based on these results, we find that protocols for networking with ESD antennas need to adapt to the varying environmental conditions. We show that the received signal strength $(R S S I)$ is a very good indicator for the success of future transmissions when using a certain antenna direction to transmit to a neighbouring node. Based on this insight, we present a simple and energyefficient mechanism to identify the best antenna direction. Its overhead is below $2 \%$ compared to a standard omni-directional transmission with a common WSN MAC layer. We achieve this performance using a train of consecutive packets quickly transmitted within the same radio activation. This mechanism can serve as a building block for adaptive protocols for WSNs with ESD antennas. 


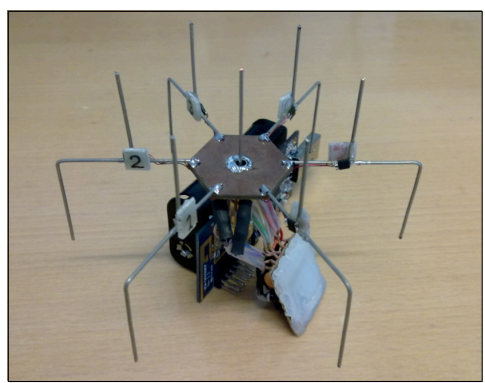

(a) SPIDA prototype, connected to a TMote Sky node

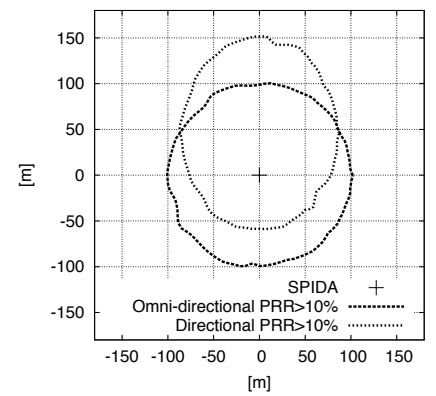

(b) SPIDA: area where the packet reception rate is larger than $10 \%$ [2]

Fig. 1: SPIDA prototype and area of directional main lobe.

Our main contributions are the following:

- We study the long-term behaviour of ESD antennas in indoor and nonLOS situations showing that the best antenna direction may change over time and that the best direction is not necessarily the same for all 802.15.4 channels as previous results suggest [9]. These results imply that protocols for networking with ESD antennas need to adapt.

- We demonstrate that an intentionally simple and efficient mechanism that selects the best antenna direction based on the RSSI of one packet for each antenna direction is sufficient to choose the best antenna direction with high probability.

Our findings provide valuable input for designers of protocols for sensor networks with ESD antennas.

The paper proceeds as follows. In the next section we present the SPIDA antenna. Section 3 discusses our methodology and experimental setup. The following sections discuss spatial, temporal and inter-channel variations. In Section 7 we present a mechanism to find the best performing antenna direction at run-time. Before concluding, we discuss related work in Section 8.

\section{Antenna Prototype}

We use a switched parasitic element antenna [10] called SPIDA, designed by Nilsson at SICS specifically for WSNs [5]. The antenna operates in the $2.4 \mathrm{GHz}$ ISM band, matching the most common WSN radio technology. In our prototype, we integrate SPIDA with a standard TMote Sky node [11]. To be compliant with the requirements of WSNs, SPIDA has a small form factor, comparable in size to the sensor node itself, as Figure 1(a) shows.

SPIDA has one central active element surrounded by six "parasitic" elements. The central element is a traditional omni-directional antenna, implemented as a quarter-wavelength whip antenna. The parasitic elements can be switched 
between ground and isolation: when grounded, they work as reflectors of radiated power; when isolated, they operate as directors of radiated power. The parasitic elements are individually controllable, yielding six possible "switches" to control the shape and direction of the antenna main lobe. They may also be all isolated: in this case, SPIDA behaves as an omni-directional antenna, which simplifies broadcasting and neighbour discovery.

The antenna gain is designed to smoothly vary as an offset circle from approximately $7 \mathrm{~dB}$ to $-4 \mathrm{~dB}$ in the horizontal plane, with the highest gain in the direction of the isolated element(s). Particularly, when the $n^{\text {th }}$ parasitic element is isolated and all other are grounded, SPIDA operates in the most directional manner, and we say that SPIDA has direction $n$. Figure 1(b) exemplifies the performance in packet reception rate $(P R R)$ with this specific configuration, based on an empirical model we derived earlier [12]. In the picture, the isolated parasitic element is directed upwards. Compared to the omni-directional SPIDA configuration, the area where at least $10 \%$ of the packets are received moves upwards and becomes slightly narrower in the orthogonal direction.

The antenna is also straightforward to manufacture, and its most expensive part is the SMA connector (about $\$ 6$ in single quantities). The cost, size, and radiation characteristics of SPIDA are therefore comparable with the state of the art in directional antennas for low-power wireless $[3,13,14]$ rendering our results of general applicability.

\section{Experimental Setup and Methodology}

To provide protocol designers with indications on the temporal behaviour of directional antennas across different channels, we need to create challenging conditions for our experiments. To this end, we deploy a small sensor network in an office environment, as depicted in Figure 2. Compared to outdoor scenarios, our setup includes both fixed (walls) and moving (people) obstacles. Moreover, several co-existing 802.11 and 802.15.4 networks operate in the same area which creates realistic conditions of interference. The nodes are also placed to create both line of sight (LOS) and non-LOS conditions across different devices and points in time.

We deploy a node equipped with the SPIDA antenna in an office of roughly 2 $\mathrm{m}$ by $4 \mathrm{~m}$. All other nodes in our setup are equipped with the standard TMote Sky microstrip inverted-F antenna, and passively act as probes to monitor the transmissions from the SPIDA node. Direction 1 of the SPIDA antenna points straight to node 4 . The two nodes are thus always in LOS conditions. Node 4 is the only node in our setup that enjoys such conditions throughout all experiments. Node 3 and node 5 on the other hand, may be in LOS conditions depending on the presence of people in the office, who may interrupt the line of sight. This is in particular true for node 3 where the LOS is interrupted when a person sits at the desk, whereas for node 5 there is rarely a person blocking LOS. Node 2 does not enjoy LOS as there are fixed obstacles that block LOS almost completely. Node 6 and 7 are in different offices than the SPIDA node. 
People sitting in these offices may further create obstacles for transmissions in addition to the walls.

To carry out the experiments, we implement small Contiki programs for both the SPIDA node and the probes. The former controls the execution of the experiments and collects the data to compute statistics. We use the IEEE 802.15.4 channel 15 as control channel, as we expected it to be the least interfered one in our setting, based on previous experiments. The processing starts by broadcasting a START message that informs the probe nodes about the beginning of an experiment. This message is not accounted for in the statistics. Next, the SPIDA node starts broadcasting dummy packets with different transmission power on channels 11, 15, 19 and 23. We chose these channels since we expect 11 and 15 to have low interference, channel 19 to have moderate interference, and channel 23 to be significantly in-

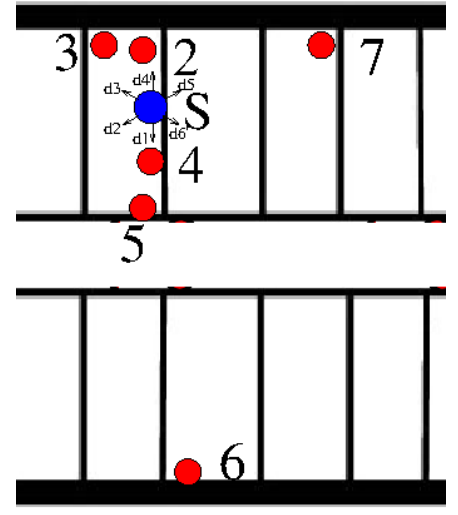

Fig. 2: Experimental setup. The node denoted with $\mathrm{S}$ is equipped with the SPIDA antenna. All others have microstrip inverted-F antennas, according to the standard TMote Sky design. terfered. The packets are sent with an inter packet transmission time of $1 / 8$ seconds. Each train of dummy packets consists of 10 packets. We did not opt for lower inter packet transmission times to avoid successive packet loss due to link burstiness [15].

After receiving the 10 th packet or when a timeout expires, the probes send back data on the received signal strength $(R S S I)$, the link quality indicator $(L Q I)$, the noise floor and the signal to noise ratio (SNR) for each received packet to the SPIDA node that stores the data in a log file for further processing. The SPIDA node also estimates the external interference on each of the channels we use by measuring a simplified form of the channel quality (CQ) metric [16]. The CQ value is computed as the fraction of RSSI samples that are above a certain threshold. For our measurements, we have set the threshold to $-77 \mathrm{dBm}$, the CC2420's default CCA threshold. The CQ value is 1 in the absence of interference. The CQ measurements confirmed our expectations on the channel quality except that it turned out that channel 15 was slightly more interfered than channel 11.

To provide a quantitative measure of the performance variations across different antenna directions, we use the statistical entropy [17] as metric. The entropy of a given random variable $\mathrm{R}$ is defined as:

$$
H(R)=\sum_{r}-p_{r} * \log \left(p_{r}\right)
$$

Therefore, the entropy is 0 whenever a random variable always takes the same value. In our case, this means that when one direction is always performing best, 


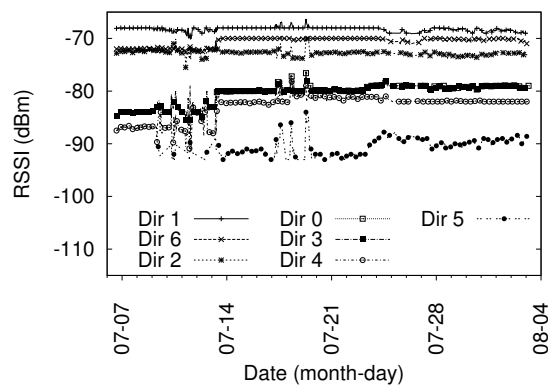

(a) Node 4: LOS conditions, TX power 5, channel 11 .

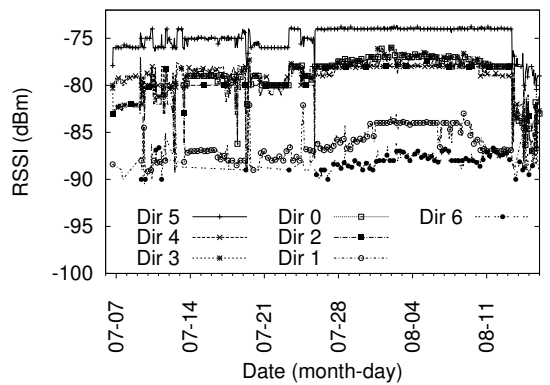

(b) Node 7: non-LOS conditions, TX power 21, channel 19 .

Fig. 3: Link performance along different antenna directions, compared to the omni-directional configuration. The direction of maximum gain provides an increase in $R S S I$ between $10 \mathrm{dBm}$ and $4 \mathrm{dBm}$.

the entropy is 0 . The maximum entropy, i.e., the highest degree of uncertainty on the outcome of a random variable, exists when the probability of the variable taking any value in its domain is equal.

We run experiments from early July until the middle of August. From mid July until the beginning of August, most of the offices are empty due to vacations, including those where we do not deploy nodes. Starting July 13, we also broadcast dummy packets in omni-directional mode, to obtain a baseline. Unfortunately, node 4 stopped working during the night of August 3rd, so results for it are available only partially.

\section{Spatial Variations}

The ability to dynamically steer the radiated power in given directions brings an additional degree of freedom to the network configuration. Protocols need to handle the possible settings depending on performance requirements. A key aspect in this regard is to understand the variability of the link performance across different antenna directions depending on the receiver's position.

Figure 3 exemplifies the RSSI link performance along different antenna directions, compared to the omni-directional configuration. We discuss two sample cases for LOS and non-LOS configurations and different TX power settings, although we obtained similar results in different scenarios as well.

Figure 3(a) shows the RSSI at node 4 when the SPIDA node is transmitting on channel 11 at TX output power 5 (around $-20 \mathrm{dBm}$ ). The SPIDA antenna is in LOS with node 4 and the parasitic element corresponding to direction 1 points directly towards it. As expected, direction 1 indeed provides the highest $R S S I$ reading at node 4 throughout the trace. The gap from the omni-directional configuration is around $10 \mathrm{dBm}$, which is significant.

Moreover, the adjacent directions 6 and 2 also yield $R S S I$ values higher than the omni-directional configuration. This is a result of the SPIDA design, whose 
main lobe in the direction of maximum gain is shaped as an offset circle, as shown in Figure 1(b). Accordingly, the remaining SPIDA directions result in average $R S S I$ values at node 4 lower than the omni-directional configuration.

As for $P R R$, with TX power 5 , node 4 receives all packets sent by the SPIDA node. Using TX power 2 (smaller than $-25 \mathrm{dBm}$ ), node 4 receives only packets that SPIDA transmits in directions 1, 2 and 6. For direction 1, the PRR is almost $99 \%$ while it is $80.8 \%$ and $62.7 \%$ for directions 6 and 2 , respectively. When SPIDA transmits in omnidirectional mode at TX power 2, node 4 does not receive any packets. This confirms the trends shown in Figure 3(a), but at lower TX power all SPIDA configurations but directions 1, 6 , and 2, fall below the radio sensitivity.

Figure 3(b) depicts the RSSI performance for node 7, which is in non-LOS conditions unlike node 4 . Particularly, packets transmitted to node 7 need to pass through at least two walls. The picture shows the RSSI readings at this node when SPIDA is transmitting on channel 19 at TX output power 21 (around $-4 \mathrm{dBm})$. Over the whole trace, packets that SPIDA transmits in direction 5 are received with the highest $R S S I$ : around 4 to $6 \mathrm{dBm}$ higher than the omnidirectional configuration. The directions 2, 3 and 4 yield a performance similar to omnidirectional. On the other hand, directions 1 and 6 shows very poor performance even though direction 6 is adjacent to the best direction 5. Most likely, multi-path effects due to non-LOS conditions make it perform worse than its counterpart direction 4 that is also adjacent to direction 5 .

In terms of $P R R$, node 7 receives almost all packets when SPIDA transmits in direction 5. Also, directions 2, 3 and 4 have a high PRR of around $97.5 \%$, while the directions with the lowest $R S S I$, direction 1 and direction 6 , also have a low PRR of $75.3 \%$ and $51.1 \%$. Based on the discussion above, we state the following:

Observation 1. Using a dynamically steerable directional antenna, the best performing antenna direction is most likely related to the receiver's physical position when nodes are in LOS conditions. In LOS, side lobes also perform well. In non-LOS conditions the best direction is not necessarily related to the physical position with respect to the sender but depends on multi-path effects.

Most protocols are unaware of this behavior, and therefore do not take advantage of it. Note that it is possible to estimate if nodes are in LOS conditions at run-time. For example, in order to estimate the distance between two sensor nodes, Pettinato et al. measure the round-trip times of ranging packets over all channels [18]. When the variance of the round-trip times across all channels is small, this is an indication that the impact of multi-path effects is low which suggests that nodes are in LOS.

\section{Temporal Variations}

As much as the link performance across different antenna directions shown in Figure 3 remains reasonably stable, moving obstacles and changing environmental conditions may make it vary over time. In this sense, the use of dynamically 


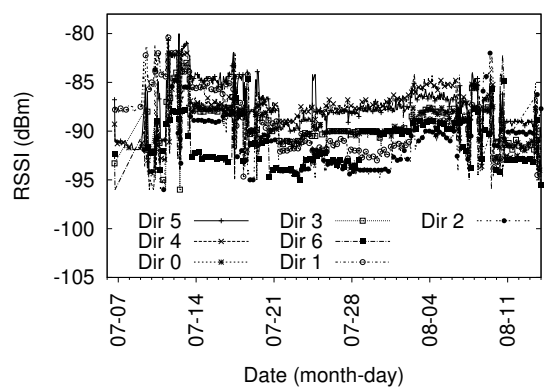

(a) Node 6: non-LOS conditions, TX power 17, channel 19 .

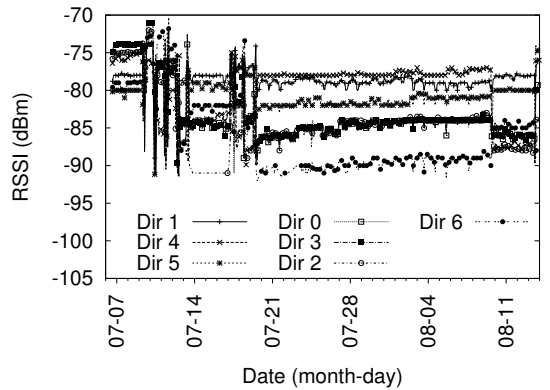

(b) Node 2: non-LOS conditions, TX power 5, channel 15 .

Fig. 4: Link performance along different antenna directions, compared to the omni-directional configuration. The best performing antenna direction changes over time.

steerable directional antennas is not different from omni-directional ones, as we discuss in the following.

Figure 4 shows two example traces of $R S S I$ readings where different antenna directions provide significantly different performance over time. We observe similar trends in different configurations as well. Figure 4(a) shows the RSSI at node 6 that is in non-LOS of the SPIDA, using TX power 17 on channel 19. For example, direction 4 is the best performing direction (in terms of $R S S I$ ) between July 20th and August 6th, but yields the worst performance in the beginning of July and some days around August 12. Direction 5 is the best direction for two smaller periods of time (July 14 to July 18 and August 11 to August 14). Moreover, direction 2 sometimes provides good performance, whereas it also results around $8 \mathrm{dBm}$ worse than direction 4 for non-negligible periods of time. Direction $5(96.7 \%)$ and direction $4(94.1 \%)$ provide the best $P R R$ over the duration of the experiment while the $P R R$ of direction 2 suffers from the period of bad performance and has the lowest average $P R R$ of all directions, slightly below $85 \%$.

The figure also demonstrates periods where the performance of given antenna directions is fairly stable and other periods where there are significant instabilities, with the best antenna configuration varying distinctly over time. The times when links are unstable correspond to the when there are persons in the offices, particularly in the one where we deploy node 6 .

These observations generally apply to non-LOS nodes independently of their distance from the SPIDA node. For example, Figure 4(b) shows the RSSI performance at node 2 , using channel 15 and TX power 5 (around $-20 \mathrm{dBm}$ ). Although the variation in the plot is not as significant as in Figure 4(a), the performance is not at all stable, especially in the early parts of the trace. Probably, the person assigned to the office where we deploy node 2 was less in his office during the duration of the experiments. While with TX power 5, the RSSI for most 


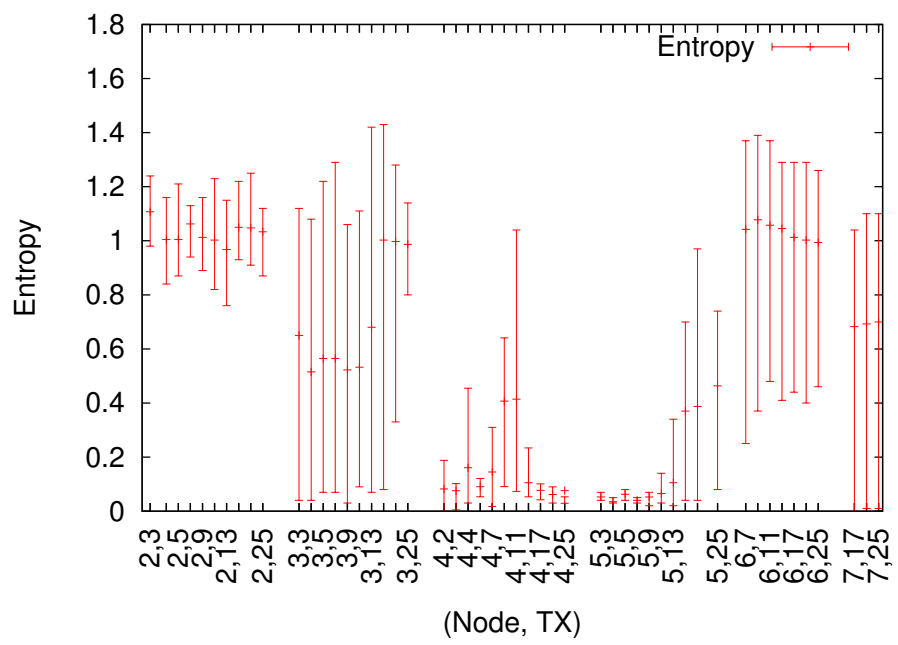

Fig. 5: Average, maximum, and minimum entropy of best performing antenna direction depending on receiver node and TX power.

directions is not close to the sensitivity threshold at the receiver, direction 6 and direction 2 lose more than $20 \%$ resp. $10 \%$ of their packets.

Based on the percentage of time a given antenna direction provides the best link performance in our experiments, we can use the entropy to cater for an aggregate indication of the temporal variations of the link performance. In our case, the maximum value of entropy would be $H=\log (6)=1.7918$. Both traces in Figure 4 thus correspond to significantly high levels of uncertainty, holding $H=1.287$ for node 6 in Figure $4(\mathrm{a})$ and $H=1.208$ for node 2 in Figure 4(b).

Figure 5 reports the average, maximum, and minimum entropy for all traces we collect, against the receiver node and TX power. The plot demonstrates that, depending on the receiver's position, the uncertainty in establishing the best performing antenna configuration may be significant. On the other hand, the LOS conditions again play a role: node 4 is among the ones with lowest entropy in the chart.

According to the findings hitherto discussed, we can claim:

Observation 2. The link performance of $\mathrm{ESD}$ antennas is time-varying. In particular, the best antenna direction may change over time.

This issue is, unfortunately, largely overlooked in the state of the art. Several existing protocols, for example, include a distinct discovery phase [8]. Our results demonstrate that after the initial discovery phase continuous monitoring and adaptation is necessary as the initial configuration may turn out to perform poorly in the long term. 


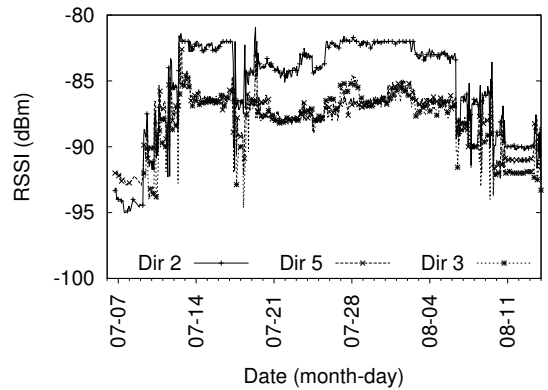

(a) Channel 11: best performing antenna direction is 2 .

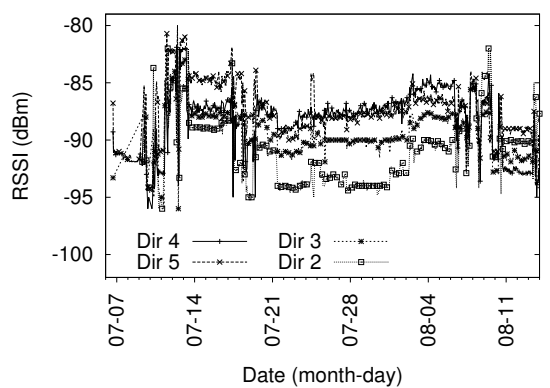

(b) Channel 19: best performing antenna direction is 4 or 5 .

Fig. 6: Link performance on different channels for node 6 at TX power 17. The best performing antenna direction depends on the channel.

\section{Inter-channel Variations}

Based on the results discussed in the previous two sections, we argue that multipath effects play a key role also depending on the specific antenna configuration. Since different IEEE 802.15.4 channels typically imply different multi-path effects, here we study the relation between this setting in 802.15.4 networks and the link performance depending on the antenna direction.

We consider as example the link performance at node 6 using different channels and TX power 17, shown in Figure 6 . Using channel 11, the best performing antenna direction is 2, as Figure 6(a) demonstrates. Direction 2 has also the highest PRR with $94.8 \%$ packets received, and remains the best antenna configuration most of the time. On channel 19, the situation is different: the best performing antenna directions are 4 and 5 . Direction 5 also corresponds to the best $P R R$ overall (96.7\%), followed by direction 4 with a $P R R$ of $93.4 \%$. Using channel 19, direction 2 performs quite poorly and, in contrast to channel 11 where it performs best, never constitutes the best antenna configuration. The $P R R$ using this direction is also the lowest $P R R$ of all possible antenna directions $(85.1 \%)$. On the contrary, using channel 11, direction 4 performs badly and achieves a PRR of only $75 \%$ : much lower than $93.4 \%$ on channel 19 . As mentioned in Section 3, node 6 is not in the same office as the SPIDA node and hence multi-path induced fading may be severe and different for different channels.

The same discussion as above applies to other nodes. For example, at node 2 , when SPIDA is configured with TX power 4, direction 5 yields a $P R R$ of $99 \%$ on channel 11 while the same direction on channel 19 is the worst configuration with a $P R R$ of only $73.3 \%$. For node 7 , when SPIDA's TX power is 17 , direction 1 and direction 5 are the best performing directions on channel 23, both with a $P R R$ of about $78 \%$, whereas on channel 11 the PRR for direction 1 is only $19 \%$.

In Figure 7 we show the average, maximum, and minimum entropy of the best performing antenna direction against receiver node and radio channel. In this case, the maximum entropy would be again $H=\log (6)=1.7918$ out of 


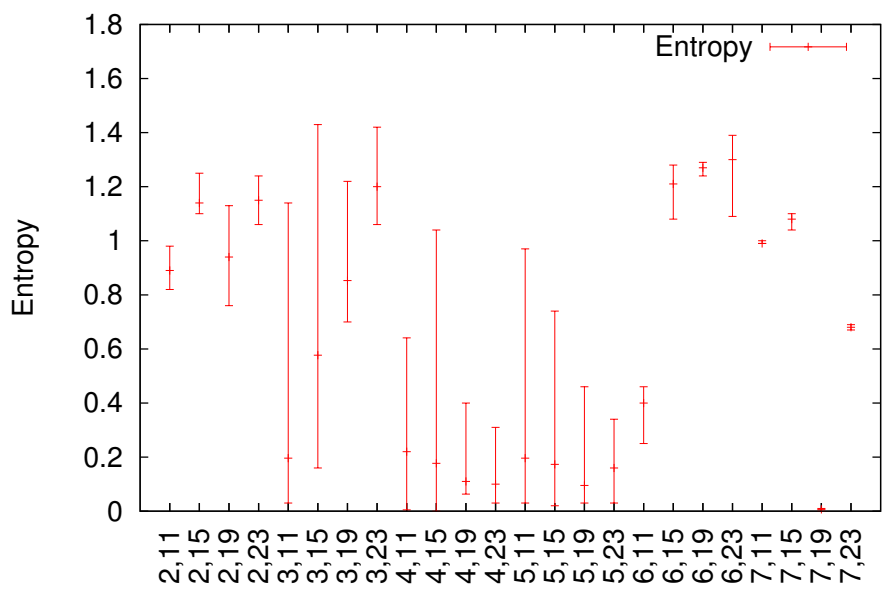

(Node, channel)

Fig. 7: Average, maximum, and minimum entropy of best performing antenna direction depending on receiver node and radio channel.

the six possible antenna directions. Two considerations emerge: i) varying the channel, the best performing antenna direction exhibits about the same degree of significant uncertainty as when varying the TX power; and ii) at some nodes, e.g., node 6 and node 7 , the degree of uncertainty appears to be remarkably consistent across different traces, which suggests that the phenomena may be independent of the specific environmental conditions during an experiment.

The discussion above leads us to the following:

Observation 3. Due to multi-path effects that behave differently on different channels, the best performing antenna configuration on a channel does not necessarily keep the same performance on a different channel.

This observation has implications for protocols using multiple channels. The main motivations for using multiple channels is to avoid external interference [19, 20]. Our results, however, imply that when switching channels, protocols cannot retain the same antenna configuration, at least when nodes are not in LOS.

\section{Taming the Added Complexity}

Notwithstanding the potential advantages of using dynamically steerable directional antennas in WSNs, the previous sections demonstrate that the use of this antenna technology may add to the complexity of current protocol designs. Indeed, the antenna's ability to steer the radiated power in given directions at run-time increases the degrees of freedom in configuring the network stack, as the specific antenna configuration plays a key role in determining the performance. Finding an efficient antenna configuration, on the other hand, requires adaptive mechanisms, as the best performing configurations are time varying and channel dependent. 
In order to capture the characteristics of low power links, sensor network protocols use link quality estimators (LQE) [21]. The goal of such an estimator is to quickly with as little energy overhead as possible predict the probability of success of future packet transmissions. While hardware-based estimators such as the RSSI, $L Q I$ and $S N R$ read values directly from the radio chip, softwarebased LQEs compute statistics over a number of transmissions [21]. For our purpose of differentiating between different directions, it would be ideal to use a hardware-based LQE that could identify the best direction with one packet only. In order to evaluate whether this is feasible we set up another experiment using the same nodes and positions described in Section 3. We run the experiment for a whole week from August 27 to September 3. As the experiment is conducted after the vacation period, there is in general more interference than during the experiments in the previous sections as confirmed by the lower CQ values on all channels. A higher degree of external interference makes link estimation more difficult.

In this experiment, the SPIDA node (after the START message) sends 101 packets in a round-robin fashion on each antenna direction, again with an inter packet transmission time of $1 / 8$ seconds. After the reception of the last packet or a timeout in case the last packet is lost, the probes return the RSSI and $L Q I$ of the first packet, as well as the number of packets received out of the probe messages number 2 to 11,2 to 51 and 2 to 101. We do this over the same four channels $(11,15,19$ and 23) with selected TX output powers. The TX output powers are selected so that all nodes have some packet loss at some TX output power. We select TX output power 2, where node 4 does not receive all packets, TX output power 3 , where nodes 2,3 and 5 lose some packets and TX output power 21 where node 6 and node 7 do not receive all packets. Based on the RSSI or $L Q I$ of the first packet, we select one direction, the one with the highest $R S S I$ resp. $L Q I$, as the estimated best direction. Since we have the packet receptions of all directions, we can then check whether this selected direction is indeed the best one. We also check if the selected direction is among the three best directions in order to see if we have been successful in avoiding bad antenna directions.

Our results are depicted in Figure 8. The graph shows that the RSSI generally performs better than the $L Q I$ for finding the best direction. Based on the RSSI of only one packet in each direction, the best direction is picked between around $85 \%$ of the time for the nodes in the same office (node 2 to node 5 ) for the next 10 packets. For the next 50 packets, this number decreases to around 65 to $75 \%$ for these nodes and is roughly the same for the next 100 packets which indicates the stability of links. For node 6 and node 7, the results are little worse but still acceptable. Remarkable, however, is that our approach avoids picking bad directions. Figure 8(a) shows that one of the best three directions is picked with a probability of more than $90 \%$ even for the nodes in the other offices (node 6 and node 7).

We can leverage the results above to build a simple mechanism to find the best performing antenna direction at run-time, as well as to adapt to the changes 


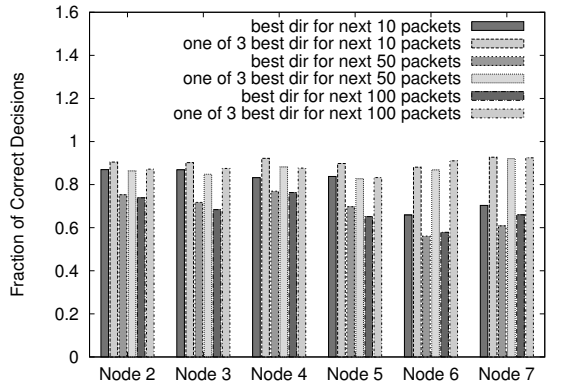

(a) RSSI as direction quality estimator.

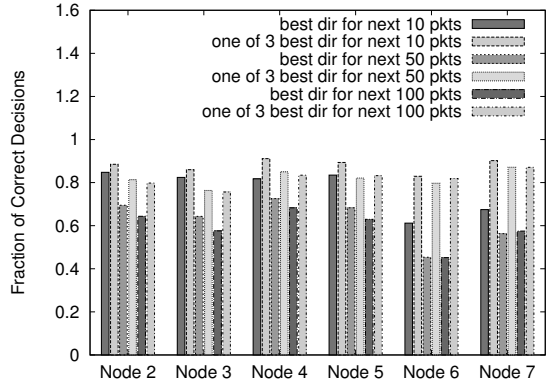

(b) $L Q I$ as link quality estimator.

Fig. 8: Performance of the direction quality estimators. The graphs show how often we pick the best direction or one of the three best directions for the next 10, 50 or 100 packets based on the RSSI or LQI of one packet only. Both RSSI and $L Q I$ perform well with $R S S I$ performing better.

in link quality that will inevitably occur. To do so, we modify the SmART strategy we proposed earlier [2].

Using SMART, every packet is quickly transmitted over all possible antenna directions, in sequence. To specify what direction is used, the transmitter appends the identifier of the active direction to the packet, encoded with a corresponding number of padding bytes. Packets can therefore be transmitted very fast, as there is no need to re-load the radio buffer: only the total packet size is changed, not the content.

At the receiver side, we measure the $R S S I$ for each received packet. Once the transmitter sweeps all directions, or upon expiration of a timeout corresponding to the maximum time required to do so, the receiver transmits back an acknowledgement carrying the identifier of the antenna direction that yields the maximum $R S S I$ value at the receiver.

The resulting schema is very cheap in terms of energy consumption. Compared to the average energy cost for transmitting one packet using ContikiMAC in the traditional way, our schema imposes only a $1.98 \%$ overhead [2]. Indeed, the bulk of the energy cost using state-of-the-art MAC protocols is elsewhere, e.g., in the strobing phase for ContikiMAC. Because of this, our schema can be used frequently, allowing protocols to keep track of the dynamics of directional links and to execute dedicated adaptation mechanisms.

\section{Related Work}

In contrast to other works that investigate link layer issues for sensor networking with directional antennas, we perform long-term indoor experiments in which we study multiple channels and also include nodes that are not placed in line of sight.

We have earlier compared the performance of the SPIDA antenna against omni-directional antennas demonstrating both that SPIDA radiates the power 
mostly in the chosen directions and that the link quality is more stable [22]. We then developed a link-layer model based on empirical measurements [12]. Also Giorgetti et al. assess the improvements in link performance but with a different prototype antenna that is designed by combining four patch antennas [13]. While this study confirms our previous results none of the studies above has looked at multiple channels, temporal variations, or non LOS placement of sensor nodes.

We investigate the long term link behaviour of non-standard antennas. Others have investigated the characteristics of low power wireless links in special environments. Ceriotti et al. have studied wireless links in a jungle [23], although mostly with short-term experiments. Long-term studies of the behaviour of lowpower wireless links are instead found in the literature for peculiar settings such as road tunnels [24], along with a comparison against more traditional settings.

Sensor and ad-hoc networking with directed antennas opens a lot of challenging research questions including neighbour discovery, medium access control, and routing [25]. For example, Felemban et al. present a TDMA-based MAC layer for sectored antennas that bootstraps with a very energy-consuming procedure for matching senders' and receivers' sectors [8]. Their approach has inspired us to investigate whether such a one-shot approach delivers the optimal configuration also over a longer time or whether the best configuration varies over time.

Our approach to dynamically select the best direction uses SMART that we have developed in the context of efficient data collection with directional antennas [2]. Incorporating the RSSI into our approach is motivated by Zuniga et al.'s study, which aims at identifying the best of a set of unreliable links [26]. They show that RSSI and $L Q I$ are better estimators than $P R R$ when only a small number of packets are used for link ranking.

As noted above also ad-hoc networking researchers have used directional antennas [25]. For example, Ramanathan et al. present a fully working system [27]. Their antennas are, however, much larger and the authors do not discuss link quality issues. Navda et al. study the performance improvements possible with directional antennas for vehicular networks access [28]. They demonstrate improved throughput and longer connectivity duration compared to omnidirectional antennas.

\section{Conclusions}

This paper has investigated the link dynamics in sensor networks with ESD antennas. We have shown that the best antenna direction, i.e., the direction that leads to the highest packet reception rate and signal strength at the receiver, is not stable but varies both over time and with the selected IEEE 802.15.4 channel. Our results confirm that previous findings that have demonstrated the dependence of angle-of-arrival on channel frequency [9] also hold for directional

transmissions with ESD antennas. Therefore, protocols for such networks need to be adaptive and have to be able to select the best antenna direction at run-time. Towards this end, we have presented a mechanism that performs this selection with very low overhead. 


\section{Acknowledgements}

This work was carried out within WISENET (Uppsala VINN Excellence Center for Wireless Sensor Networks) funded by VINNOVA, Uppsala University, Banverket, CRL Sweden, FOI, Imego, JonDeTech, Pricer, SenseAir, SICS, TNTElektronik, TermoSense, VTT, Upwis, AAC Microtec, and WiseNet Holding. The work has been partially supported by the CONET NoE. Thanks to Martin Nilsson who designed the SPIDA antenna. and to our shepherd Tarek Abdelzaher for insightful comments.

\section{References}

1. J. Ko, J. Eriksson, N. Tsiftes, S. Dawson-Haggerty, M. Durvy, JP Vasseur, A. Terzis, A. Dunkels, and D. Culler. Beyond Interoperability: Pushing the Performance of Sensornet IP Stacks. In ACM SenSys, Seattle, USA, November 2011.

2. L. Mottola, T. Voigt, F. Österlind, G. P. Picco, and A. Quartulli. Electronicallyswitched directional antennas for low-power wireless networks: A prototype-driven evaluation. Technical report, Swedish Institute of Computer Science, 2012.

3. R.R. Choudhury, T. Ueda, J. Bordim, and N.H. Vaidya. Beamnet: An ad hoc network testbed using beamforming antennas. In Vehicular Technology Conference, 2005.

4. E. Felemban, R. Murawski, E. Ekici, S. Park, K. Lee, J. Park, and Z. Hameed. Sand: Sectored-antenna neighbor discovery protocol for wireless networks. In SECON 2010, June 2010.

5. M. Nilsson. Directional antennas for wireless sensor networks. In Scandinavian Workshop on Wireless Adhoc Networks, 2009.

6. F. Viani, L. Lizzi, M. Donelli, D. Pregnolato, G. Oliveri, and A. Massa. Exploitation of parasitic smart antennas in wireless sensor networks. Technical Report DISI-11-101, University of Trento, Italy, 2011.

7. R. Vilzmann and C. Bettstetter. A survey on MAC protocols for ad hoc networks with directional antennas. EUNICE 2005: Networks and Applications Towards a Ubiquitously Connected World, 196(1), 2006.

8. E. Felemban, S. Vural, R. Murawski, E. Ekici, K. Lee, Y. Moon, and S. Park. Samac: A cross-layer communication protocol for sensor networks with sectored antennas. IEEE Transactions on Mobile Computing, 9(8):1072-1088, 2010.

9. Yongwei Zhang, Anthony K. Brown, Wasim Q. Malik, and David J. Edwards. High resolution 3-d angle of arrival determination for indoor uwb multipath propagation. IEEE Transactions on Wireless Communications, 7(8):3047-3055, 2008.

10. D.V. Thiel and S. Smith. Switched parasitic antennas for cellular communications. Artec House, London, 2002.

11. J. Polastre, R. Szewczyk, and D. Culler. Telos: Enabling ultra-low power wireless research. In Proceedings of The Fourth International Symposium on Information Processing in Sensor Networks (IPSN'05), Los Angeles, CA, USA, April 2005.

12. B.S. Geletu, L. Mottola, T. Voigt, and F. Österlind. Poster abstract: Modeling an electronically switchable directional antenna for low-power wireless networks. In IPSN, 2011.

13. G. Giorgetti, A. Cidronali, S. Gupta, and G. Manes. Exploiting low-cost directional antennas in $2.4 \mathrm{ghz}$ ieee 802.15.4 wireless sensor networks. In European Microwave Week (EuMW'07), pages 18-21, Munich, Germany, October 2007. 
14. F. Viani, L. Lizzi, M. Donelli, D. Pregnolato, G. Oliveri, and A. Massa. Exploitation of parasitic smart antennas in wireless sensor networks. Journal of Electromagnetic Waves and Applications, 24(7):993-1003, 2010.

15. K. Srinivasan, M. Kazandjieva, S. Agarwal, and P. Levis. The beta factor: Measuring wireless link burstiness. In Proceedings of the Sixth ACM Conference on Networked Embedded Sensor Systems (SenSys'08), Raleigh, NC, USA, November 2008.

16. C. Noda, S. Prabh, M. Alves, C. Boano, and T. Voigt. Quantifying the channel quality for interference-aware wireless sensor networks. ACM SIGBED Review, 8(4):43-48, nov 2011.

17. T.M. Cover, J.A. Thomas, J. Wiley, et al. Elements of information theory, volume 6. Wiley Online Library, 1991.

18. P. Pettinato, N. Wirström, J. Eriksson, and T. Voigt. Multi-channel two-way time of flight sensor network ranging. In European Conference on Wireless Sensor Networks (EWSN), Trento, Italy, February 2012.

19. J. Borms, K. Steenhaut, and B. Lemmens. Low-overhead dynamic multi-channel mac for wireless sensor networks. In European Conference on Wireless Sensor Networks (EWSN), Coimbra, Portugal, February 2010.

20. L. Tang, Y. Sun, O. Gurewitz, and D. Johnson. Em-mac: a dynamic multichannel energy-efficient mac protocol for wireless sensor networks. In Proceedings of the Twelfth ACM International Symposium on Mobile Ad Hoc Networking and Computing, 2011.

21. N. Baccour, A. Koubaa, L. Mottola, M. Zuinga, H. Youssef, C.A. Boano, and M. Alves. Radio link quality estimation in wireless sensor networks: a survey. ACM Transactions on Sensor Networks (TOSN), 8(4), 2012.

22. E. Öström, L. Mottola, and T. Voigt. Evaluation of an electronically switched directional antenna for real-world low-power wireless networks. In Workshop on Real-World Wireless Sensor Networks (REALWSN), Colombo, Sri Lanka, December 2010 .

23. M. Ceriotti, M. Chini, A. Murphy, G. Picco, F. Cagnacci, and B. Tolhurst. Motes in the jungle: Lessons learned from a short-term wsn deployment in the ecuador cloud forest. In Workshop on Real-World Wireless Sensor Networks (REALWSN), Colombo, Sri Lanka, December 2010.

24. L. Mottola, G. Picco, M. Ceriotti, S. Gună, and A. Murphy. Not all wireless sensor networks are created equal: A comparative study on tunnels. ACM Trans. Sen. Netw., 7(2), September 2010.

25. H.N. Dai, K.W. Ng, M. Li, and M.Y. Wu. An overview of using directional antennas in wireless networks. International Journal of Communication Systems, November 2011.

26. M. Zuniga, I. Irzynska, J. Hauer, T. Voigt, C. Boano, and K. Römer. Link quality ranking: Getting the best out of unreliable links. In 7th IEEE International Conference on Distributed Computing in Sensor Systems (DCOSS), Barcelona, Spain, June 2011.

27. R. Ramanathan, J. Redi, C. Santivanez, D. Wiggins, and S. Polit. Ad hoc networking with directional antennas: a complete system solution. Selected Areas in Communications, IEEE Journal on, 23(3):496-506, 2005.

28. V. Navda, A.P. Subramanian, K. Dhanasekaran, A. Timm-Giel, and S. Das. Mobisteer: using steerable beam directional antenna for vehicular network access. In Proceedings of the 5th international conference on Mobile systems, applications and services (MobiSys), San Juan, Puerto Rico, June 2007. 